\title{
A Digital Lighting Function for Strong 26-Surfaces
}

\author{
R. Ayala ${ }^{1}$, E. Domínguez ${ }^{2}$, A.R. Francés ${ }^{2}$, A. Quintero $^{1}$ \\ 1 Dpt. de Geometría y Topología. Facultad de Matemáticas. Universidad de Sevilla. \\ Apto. 1160. E-41080 - Sevilla. Spain. \\ e-mail: quintero@cica.es \\ 2 Dpt. de Informática e Ingeniería de Sistemas. Facultad de Ciencias. \\ Universidad de Zaragoza. E-50009 - Zaragoza. Spain. \\ e-mail: ccia@posta.unizar.es
}

\begin{abstract}
The goal of this paper is to generalize the notion of lighting function given in [3] in order to integrate strong 26-surfaces [5] into our framework for digital topology. In particular, the continuous analogue for strong 26-surfaces introduced in [10] is extended for arbitrary objects.
\end{abstract}

Keywords: Lighting function, digital surface, strong 26-surface.

\section{Introduction}

In a series of papers $1,2,3$ we introduced an approach to the notion of digital space within a new framework for digital topology. This framework is presented as a multilevel architecture which provides a link between a device level, where the discrete nature of digital objects is represented, and an Euclidean space. The most elaborate notion of digital space was given in 3] by introducing the notion of lighting function, which intends to formalize the idea of "continuous perception" that an observer may take on digital objects. In this way, a digital space is not only determined by a device model but also by a lighting function defined on it. In some sense, such a function is providing a method to construct a continuous analogue from each digital object, which is actually the "continuous perception" we are considering on that object.

Based on the idea of "face membership rule" from Kovalevsky 9], lighting functions were originally defined through a set of four axioms, allowing a wide family of "continuous perceptions" used in literature. In fact, for all $\alpha, \beta \in$ $\{6,18,26\}$ there exist lighting functions providing the $(\alpha, \beta)$-connectedness, defined on $\mathbb{Z}^{3}$ within the graph-based approach to digital topology [8]; and, moreover, $(\alpha, \beta)$-surfaces [11,7] are also found as surfaces in the corresponding digital spaces, for $(\alpha, \beta) \neq(6,6)$. However these four axioms are not general enough to replicate the "continuous perception" associated with strong 26-surfaces in 5 .

Our main goal in this paper is to introduce a set of axioms for lighting functions more general than that in [3. Def. 1]. These new axioms allow us to find a suitable digital space whose surfaces are exactly the set of strong 26-surfaces (Th. 5). Several consequences are derived from this result. Firstly, 
in [10] Malgouyres and Bertrand define a continuous analogue only for strong 26-surfaces, which is now extended for arbitrary objects in our architecture. Moreover, results (e.g., the Digital Jordan-Brower Theorem) which have been proved in our framework with fully generality hold for strong 26-surfaces without further proof. Finally, our main result (Th. 5) provides a proof for possibly infinite strong 26-surfaces of the local characterization given in [10, Th. 6] only for finite strong 26-surfaces.

These generalized lighting functions are introduced in Section 2, where we also recall some of the basic aspects of our framework, as well as some notations and results used in this paper. In Section 3 we give the appropriate digital space for which digital surfaces coincide with strong 26-surfaces. This is proved in Sections 4 and 5 .

\section{The multilevel architecture}

As in 123 the first level of a digital space, called the device model, is used to represent the spatial layout of pixels, which are represented by the $n$-cells of a homogeneously $n$-dimensional locally finite polyhedral complex $K$. Namely, $K$ is a complex of convex cells (polytopes) such that each cell is face of a finite number (non-zero) of $n$-cells. If $\gamma$ is a face of $\sigma$ we shall write $\gamma \leq \sigma$, and $\gamma<\sigma$ if in addition $\gamma \neq \sigma$. If $|K|$ denotes the underlying polyhedron of $K$, a centroid-map is a map $c: K \rightarrow|K|$ such that $c(\sigma)$ belongs to the interior (as a cell) of $\sigma$; that is, $c(\sigma) \in \sigma-\partial \sigma$, where $\partial \sigma=\cup\{\gamma ; \gamma<\sigma\}$ stands for the boundary of $\sigma$. The set of all $n$-cells of $K$ will be denoted by $\operatorname{cell}_{n}(K)$. Given a device model $K$, a digital object in $K$ is a subset of the set $\operatorname{cell}_{n}(K)$ of $n$-cells in $K$.

In this paper we will deal with the device model $R^{n}$, called the standard cubical decomposition of the $n$-dimensional Euclidean space $\mathbb{R}^{n}$. This device model $R^{n}$ is the complex determined by the collection of unit $n$-cubes in $\mathbb{R}^{n}$ whose edges are parallel to the coordinate axes and whose centers are in the set $Z^{n}$. The centroid-map we will consider in $R^{n}$ associates to each cube $\sigma$ its barycenter $c(\sigma)$. In particular, if $\operatorname{dim} \sigma=n$ then $c(\sigma) \in \mathbb{Z}^{n}$, where $\operatorname{dim} \sigma$ stands for the dimension of $\sigma$. So that, every digital object $O$ in $R^{n}$ can be identified with a subset of points in $Z^{n}$. Henceforth we shall use this identification without further comment.

Now we are ready to introduce the notion of weak lighting function generalizing lighting functions in [3]. For this we need the following notation.

Given a cell $\alpha \in K$ and a digital object $O \subseteq \operatorname{cell}_{n}(K)$, the star of $\alpha$ in $O$ and the extended star of $\alpha$ in $O$ are respectively the digital objects $\operatorname{st}_{n}(\alpha ; O)=$ $\{\sigma \in O ; \alpha \leq \sigma\}$ and $\operatorname{st}_{n}^{*}(\alpha ; O)=\{\sigma \in O ; \alpha \cap \sigma \neq \emptyset\}$. Notice that if $\operatorname{dim} \alpha=0$ then $\operatorname{st}_{n}(\alpha ; O)=\operatorname{st}_{n}^{*}(\alpha ; O)$ for any digital object $O$ in $K$. The support of $O$, $\operatorname{supp}(O)$, is the set of all cells $\alpha \in K$ such that $\alpha=\cap\left\{\sigma ; \sigma \in \operatorname{st}_{n}(\alpha ; O)\right\}$. To ease the writing, when the digital object is the whole set cell ${ }_{n}(K)$ we shall write $\operatorname{supp}(K), \operatorname{st}_{n}(\alpha ; K)$ and $\operatorname{st}_{n}^{*}(\alpha ; K)$ instead of $\operatorname{supp}\left(\operatorname{cell}_{n}(K)\right), \operatorname{st}_{n}\left(\alpha ; \operatorname{cell}_{n}(K)\right)$ and $\operatorname{st}_{n}^{*}\left(\alpha ; \operatorname{cell}_{n}(K)\right)$, respectively. Finally, we shall write $\mathcal{P}(A)$ for the family of all subsets of a given set $A$. 


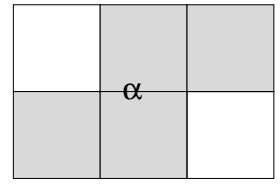

$O$

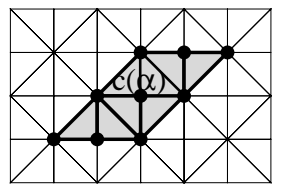

$\mathcal{A}_{O}$

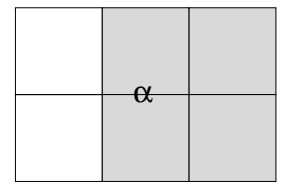

$O^{\prime}$

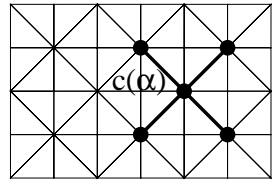

$\mathcal{A}_{O^{\prime}}$

Fig. 1.The w.l.f. $f$ in Example 1 does not satisfy property (F3).

Definition 1. Given a device model $K$, a function $f: \mathcal{P}\left(\operatorname{cell}_{n}(K)\right) \times K \rightarrow\{0,1\}$ is said to be a weak lighting function (w.l.f.) on $K$ if it verifies the following five properties for all $O \in \mathcal{P}\left(\operatorname{cell}_{n}(K)\right)$ and $\alpha \in K$.

1. If $\alpha \in O$ then $f(O, \alpha)=1$.

2. If $\alpha \notin \operatorname{supp}(O)$ then $f(O, \alpha)=0$.

3. $f(O, \alpha) \leq f\left(\operatorname{cell}_{n}(K), \alpha\right)$.

4. $f(O, \alpha)=f\left(s t_{n}^{*}(\alpha ; O), \alpha\right)$.

5. Let $O^{\prime} \subseteq O$ and $\alpha \in K$ such that $\operatorname{st}_{n}(\alpha ; O)=\operatorname{st}_{n}\left(\alpha ; O^{\prime}\right), f\left(O^{\prime}, \alpha\right)=0$ and $f(O, \alpha)=1$. Then, the set of cells $\alpha\left(O^{\prime} ; O\right)=\left\{\beta<\alpha ; f\left(O^{\prime}, \beta\right)=0\right.$, $f(O, \beta)=1\}$ is not empty and connected in $\partial \alpha$. Moreover, if any digital object $\bar{O}$ is such that $O \subseteq \bar{O}$, then $f(\bar{O}, \beta)=1$ for every $\beta \in \alpha\left(O^{\prime} ; O\right)$.

Properties (1), (2) and (3) already appeared in [3] as (F2), (F1) and (F4) respectively; however properties (4) and (5) replace to property

$$
f(O, \alpha)=f\left(\operatorname{st}_{n}(\alpha ; O), \alpha\right)
$$

in [3]. As the equality $\operatorname{st}_{n}(\alpha ; O)=\operatorname{st}_{n}\left(\alpha ; \operatorname{st}_{n}^{*}(\alpha ; O)\right)$ holds for every cell $\alpha \in K$ and object $O \subseteq \operatorname{cell}_{n}(K)$, it is readily checked that property (F3) implies property (4). Moreover, if (F3) holds then no cell in $K$ satisfies all hypothesis in (5). Hence (F3) also implies property (5). So that, w.l.f.'s generalize lighting functions in [3]. Next example shows that the class of w.l.f.'s strictly contains all lighting functions.

Example 1. Let $f$ be the w.l.f. defined on $R^{2}$ by $f(O, \alpha)=1$ if and only if: (a) $\operatorname{dim} \alpha=2$ and $\alpha \in O$; (b) $\operatorname{dim} \alpha=0$ and $\alpha \in \operatorname{supp}(O)$; (c) $\operatorname{dim} \alpha=1$ and one of the two following conditions holds:

(c1) $\mathrm{st}_{2}^{*}(\alpha ; O)=\mathrm{st}_{2}^{*}\left(\alpha ; R^{2}\right)$

(c2) $\alpha \in \operatorname{supp}(O)$ and there exist $\sigma, \tau \in \operatorname{st}_{2}^{*}\left(\alpha ; R^{2}\right)-O$ such that $\sigma \cap \tau=\emptyset$. In order to check that $f$ does not satisfy property (F3), one observes that for the digital objects $O$ and $O^{\prime}$ and the 1-cell $\alpha$ in Fig. 11 the equality $\operatorname{st}_{2}(\alpha ; O)=$ st $_{2}\left(\alpha ; O^{\prime}\right)$ holds; however the definition of $f$ yields that $f(O, \alpha)=1$ while $f\left(O^{\prime}, \alpha\right)=0$.

As in [3], a digital space is defined as a pair $(K, f)$, where $K$ is a device model and $f$ is a weak lighting function on $K$. Now, given a digital object $O$ in $(K, f)$ its levels are defined as follows.

The device level of $O$ is the pair $\left(K(O), f_{O}\right)$, where $K(O)=\{\alpha \in K ; \alpha \leq \sigma$, $\sigma \in O\}$ is the subcomplex of $K$ induced by the cells in $O$, and $f_{O}$ is the restriction 
of $f$ to the set $\mathcal{P}(O) \times K(O)$. In general, this pair is not a digital space because $f_{O}$ does not satisfy property $(3)$ in Definition 1 however, $f_{O}$ turns to be a w.l.f. if it is redefined as $f_{O}\left(O^{\prime}, \alpha\right)=f(O, \alpha) f\left(O^{\prime}, \alpha\right)$, for $O^{\prime} \subseteq O$ and $\alpha \in K(O)$.

The logical level of $O$ is an undirected graph, $\mathcal{L}_{O}^{f}$, whose vertices are the centroids of $n$-cells in $O$ and two of them $c(\sigma), c(\tau)$ are adjacent if there exists a common face $\alpha \leq \sigma \cap \tau$ such that $f(O, \alpha)=1$.

The conceptual level of $O$ is the digraph $\mathcal{C}_{O}^{f}$ whose vertices are the centroids $c(\alpha)$ of all cells $\alpha \in K$ with $f(O, \alpha)=1$, and its directed edges are $(c(\alpha), c(\beta))$ with $\alpha<\beta$.

The simplicial analogue of $O$ is the order complex $\mathcal{A}_{O}^{f}$ associated to the digraph $\mathcal{C}_{O}^{f}$. That is, $\left\langle x_{0}, x_{1}, \ldots, x_{m}\right\rangle$ is an $m$-simplex of $\mathcal{A}_{O}^{f}$ if $x_{0}, x_{1}, \ldots, x_{m}$ is a directed path in $\mathcal{C}_{O}^{f}$. This simplicial complex defines the simplicial level for the object $O$ in the architecture and, finally, the continuous level is represented by the underlying polyhedron $\left|\mathcal{A}_{O}^{f}\right|$ of $\mathcal{A}_{O}^{f}$. This polyhedron is called the continuous analogue of $O$.

For the sake of simplicity, we will usually drop " $f$ " from the notation of the levels of an object. Moreover, for the whole object $\operatorname{cell}_{n}(K)$ we will simply write $\mathcal{L}_{K}, \mathcal{C}_{K}$ and $\mathcal{A}_{K}$ for its levels.

Next we recall the notion of connectedness given in 3 . Let $O$ and $O^{\prime}$ be two disjoint digital objects in a digital space $(K, f)$. Two distinct $n$-cells $\sigma, \tau \in O$ are said to be $O^{\prime}$-adjacent in $O$ if there exists a common face $\alpha \leq \sigma \cap \tau$ such that $f\left(O^{\prime}, \alpha\right)=0$ and $f\left(O \cup O^{\prime}, \alpha\right)=1$. An $O^{\prime}$-path in $O$ from $\sigma$ to $\tau$ is a finite sequence $\left\{\sigma_{i}\right\}_{i=0}^{m} \subseteq O$ such that $\sigma_{0}=\sigma, \sigma_{m}=\tau$ and $\sigma_{i-1}$ is $O^{\prime}$-adjacent in $O$ to $\sigma_{i}$, for $i=1, \ldots, m$. Then, a digital object $O$ will be said $O^{\prime}$-connected if for any pair of $n$-cells $\sigma, \tau \in O$ there exists an $O^{\prime}$-path in $O$ from $\sigma$ to $\tau$. And finally, an object $C \subseteq O$ is an $O^{\prime}$-component of $O$ if for any pair $\sigma, \tau \in C$ there exists an $O^{\prime}$-path in $O$ from $\sigma$ to $\tau$ and none element in $C$ is $O^{\prime}$-adjacent in $O$ to some element of $O-C$. Observe that any $O^{\prime}$-component is $O^{\prime}$-connected itself.

Given a digital object $O$ in the digital space $(K, f)$ the previous definitions provide an entire family of notions of connectedness for $O$ in relation to another object $O^{\prime}$, when $O^{\prime}$ is allowed to range over the set of all subsets of $\operatorname{cell}_{n}(K)-O$. The extreme cases, when $O^{\prime}=\emptyset$ and $O^{\prime}=\operatorname{cell}_{n}(K)-O$, represent the connectedness of the digital object $O$ itself and the connectedness of $O$ as the complement of $\operatorname{cell}_{n}(K)-O$, respectively. Theorem 2 shows how these notions of connectedness are stated at each level of our architecture. Below, given two subcomplexes $L_{1}$ and $L_{2}$ of a simplicial complex $L$, the simplicial complement of $L_{2}$ in $L_{1}$ will be denoted by $L_{1} \backslash L_{2}=\left\{\alpha \in L_{1} ; \alpha \cap\left|L_{2}\right|=\emptyset\right\}$.

Theorem 2. Let $O$ and $O^{\prime}$ be two disjoint digital objects in a digital space. The family $\mathcal{F}$ of $O^{\prime}$-components of $O$ can be described in any of the following ways

1. Conceptual level: $\mathcal{F}=\left\{O_{G}\right\}$, where $O_{G}=\{\sigma \in O ; c(\sigma)$ is a vertex of $G\}$, and $G$ ranges over the family of components of the digraph $\mathcal{C}_{O \cup O^{\prime}} \backslash \mathcal{C}_{O^{\prime}}$.

2. Simplicial level: $\mathcal{F}=\left\{O_{A}\right\}$, where $O_{A}=\{\sigma \in O ; c(\sigma) \in A\}$, and $A$ ranges over the family of components of the simplicial complement $\mathcal{A}_{O \cup O^{\prime}} \backslash \mathcal{A}_{O^{\prime}}$.

3. Continuous level: $\mathcal{F}=\left\{O_{X}\right\}$, where $O_{X}=\{\sigma \in O ; c(\sigma) \in X\}$, and $X$ ranges over the family of components of the space $\left|\mathcal{A}_{O \cup O^{\prime}}\right|-\left|\mathcal{A}_{O^{\prime}}\right|$. 
The proof of this result follows the same sketch given in [3], using next Lemma to prove part (1).

Lemma 3. Let $O, O^{\prime}$ be two disjoint objects in $K$ and $\alpha \in K$ any cell such that $f\left(O \cup O^{\prime}, \alpha\right)=1, f\left(O^{\prime}, \alpha\right)=0$ and $s t_{n}\left(\alpha ; O \cup O^{\prime}\right)=s t_{n}\left(\alpha ; O^{\prime}\right)$. Then there exists $\beta<\alpha$ such that $f\left(O \cup O^{\prime}, \beta\right)=1, f\left(O^{\prime}, \beta\right)=0$ and $\operatorname{st}_{n}\left(\beta ; O \cup O^{\prime}\right) \neq \operatorname{st}_{n}\left(\beta ; O^{\prime}\right)$. Moreover, if $\beta^{\prime}<\alpha$ also satisfies the above properties then there exists a path $\left\{c\left(\gamma_{i}\right)\right\}_{i=0}^{2 m}$ in $\mathcal{C}_{O \cup O^{\prime}} \backslash \mathcal{C}_{O^{\prime}}$ from $c(\beta)$ to $c\left(\beta^{\prime}\right)$ such that $\gamma_{2 j-1} \in O, 1 \leq j \leq m$.

We finish this Section giving an intuitive motivation about the replacement of property (F3) in the definition of lighting functions by properties (4) and (5) given for w.l.f.'s. Properties (F3) and (4) state that whether a cell $\alpha$ is lighted for a given object $O$ depends on a 'digital neighbourhood' $N(\alpha ; O)$ of $\alpha$ in $O$. In property (F3) $N(\alpha ; O)$ is chosen to be $\operatorname{st}_{n}(\alpha ; O)$; i.e., the smallest neighbourhood of $\alpha$ in $O$. However the continuous analogue $\left|\mathcal{A}_{\mathrm{st}_{n}(\alpha ; O)}\right|$ needs not to be, in general, a 'continuous neighbourhood' of the centroid $c(\alpha)$ in $\left|\mathcal{A}_{O}\right|$. Due to this, $N(\alpha ; O)$ is required to be $\operatorname{st}_{n}^{*}(\alpha ; O)$ in property $(4)$. With this choice the centroids of two isolated $n$-cells $\sigma_{1}, \sigma_{2} \in \operatorname{cell}_{n}(K)-O, \sigma_{1} \cap \sigma_{2}=\emptyset$, can belong to the same component of the continuous space $\left|\mathcal{A}_{K}\right|-\left|\mathcal{A}_{O}\right|$ even though $\sigma_{1}$ and $\sigma_{2}$ are each one completely surrounded by $n$-cells in $O$. Thus, our continuous analogue would not provide the right representation of the continuous perception we take on digital objects. So, we require in addition axiom (5) to prevent this undesirable property.

\section{Main results}

In the multilevel architecture given in Section 2 the continuous analogue provides the "continuous perception" of digital objects. Thus, the following definition arises naturally. A digital object $S$ in a digital space $(K, f)$ is said to be a digital manifold if its continuous analogue $\left|\mathcal{A}_{S}\right|$ is a combinatorial manifold without boundary. In case $S$ is a digital surface (2-manifold) in $\left(R^{3}, f\right)$ we say that $S$ is an $f$-surface.

In this section we state our main result. Namely, we find a digital space for which an object is a digital surface if and only if it is a strong 26-surface in the sense of Bertrand and Malgouyres [5]. This digital space is defined on the device model $R^{3}$, the standard cubical decomposition of the Euclidean 3-space $\mathbb{R}^{3}$, by the w.l.f. $f^{B M}$, which is given by $f^{B M}(O, \alpha)=1$ if and only if: (a) $\operatorname{dim} \alpha=3$ and $\alpha \in O$; (b) $\operatorname{dim} \alpha=0,2$ and $\alpha \in \operatorname{supp}(O)$; (c) $\operatorname{dim} \alpha=1$ and one of the following conditions holds:

(c1) $\operatorname{st}_{3}\left(\alpha ; R^{3}\right) \subseteq O$

(c2) $\alpha \in \operatorname{supp}(O)$ and $\operatorname{st}_{3}^{*}(\alpha ; O)=\operatorname{st}_{3}(\alpha ; O)$

(c3) $\alpha \in \operatorname{supp}(O)$ and there exist $\sigma, \tau \in \operatorname{st}_{3}^{*}(\alpha ; O)$ with $\sigma \cap \tau=\emptyset$.

Remark 4. According to [3, Def. 11], it is not difficult to prove that the w.l.f. $f^{B M}$ provides the $(26,6)$-connectedness. That is, given a digital object $O$ in $\left(R^{3}, f^{B M}\right)$, the components of $O$ are exactly the 26-components of $O$; and moreover, $O^{\prime}$ is a 6 -component of $\operatorname{cell}_{3}\left(R^{3}\right)-O$ if and only if it is an $O$-component.

Our main result is now stated as follows. 
Theorem 5. A connected object $S$ in the digital space $\left(R^{3}, f^{B M}\right)$ is an $f^{B M_{-}}$ surface if and only if $S$ is a strong 26-surface.

In [10] Malgouyres and Bertrand characterize strong 26-surfaces within the family of strongly separating objects. We shall use this characterization in the proof of our Theorem 5 rather than the original definition of strong 26-surfaces, which is given in terms of strong homotopy [5]. For this we need some more notation from [10]. Here we use the identification of the grid $Z^{3} \subseteq \mathbb{R}^{3}$ with the set $\operatorname{cell}_{3}\left(R^{3}\right)$ of 3 -cells in $R^{3}$.

Given a digital object $O$ in $R^{3}$ let $N_{k}(\sigma ; O)$ denote the set of 3-cells $\tau \in O$ $(\sigma \neq \tau)$ which are $k$-adjacent to $\sigma(k=6,18,26)$, and let $G_{6}(\sigma ; O)$ be the set of 3 -cells $\tau \in N_{26}(\sigma ; O)$ such that there exists a 6 -path in $\operatorname{st}_{3}^{*}(\sigma ; O)$ from $\sigma$ to $\tau$ of length less than or equal to 2 . If $N_{k}(\sigma ; O) \neq \emptyset$ we say that $\sigma$ is $k$-adjacent to $O$. Observe that $N_{26}(\sigma ; O)=\operatorname{st}_{3}^{*}(\sigma ; O)-\{\sigma\}$. Finally, we recall that an object $O$ is said to be strongly separating if $Z^{3}-O$ has two 6-components and moreover each $\sigma \in O$ is 6-adjacent to both components (see [5]).

Definition 6. ([10, Defs. 6 and 7]) Let $S$ be a 26-connected object in $Z^{3}$. Then $S$ is said to be a near strong 26-surface if the following four properties hold for all $\sigma \in S$.

1. $N_{26}\left(\sigma ; Z^{3}-S\right)$ has exactly two 6-components $A_{1}^{\sigma}$ and $A_{2}^{\sigma}$ which are 6 adjacent to $\sigma$.

2. $G_{6}\left(\sigma ; \mathbb{Z}^{3}-S\right)$ has exactly two 6-components.

3. For each $\tau \in N_{26}(\sigma ; S)$ the sets $N_{26}\left(\tau ; A_{1}^{\sigma}\right)$ and $N_{26}\left(\tau ; A_{2}^{\sigma}\right)$ are non-empty.

4. For each $\tau \in N_{6}(\sigma ; S)$ the sets $G_{6}\left(\sigma ; A_{1}^{\sigma} \cup\{\tau\}\right)$ and $G_{6}\left(\sigma ; A_{2}^{\sigma} \cup\{\tau\}\right)$ are 6-connected.

Then, strong 26-surfaces are characterized as follows.

Theorem 7. ([10, Th. 4]) Let $S$ be a 26-connected strongly separating object in $Z^{3}$. Then $S$ is a strong 26-surface if and only if it is a near strong 26-surface.

Moreover, in [10] it is also proved

Proposition 8. ([10, Pr. 1]) Any strong 26-surface is strongly separating.

In the rest of the paper we will derive Theorem 5 from Theorem 7 and Proposition 8 as follows. In Section 4 we prove that any near strong 26 -surface is an $f^{B M}$-surface; and then, in Section 5 , we show that any $f^{B M}$-surface is both a near strong 26 -surface and strongly separating.

It is worth pointing out that, although the notion of near strong 26 -surface is local, the characterization given in Theorem 7 is not completely local because the notion of strongly separating is a global one. To obtain a local characterization of strong 26-surfaces it remains to prove that any near strong 26-surface is strongly separating. This is done in [10, Th. 6] for the class of finite strong 26-surfaces. As a consequence of our main result (Th. 5) this local characterization is now extended to possibly infinite strong 26-surfaces. Namely,

Theorem 9. (Extension of Malgouyres-Bertrand Theorem) Let $S$ be a 26-connected object in $Z^{3}$. Then $S$ is a strong 26-surface if and only if it is a near strong 26-surface. 


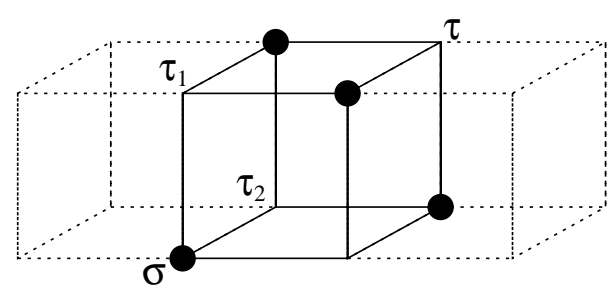

(a)

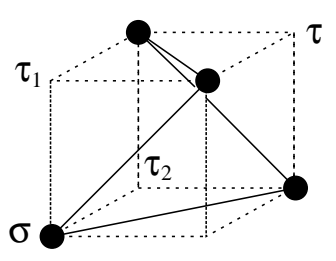

(b)

Fig. 2. A non-simple maximal elementary cube and its canonical cycle.

\section{Near strong 26-surfaces are $f^{B M}$-surfaces}

In 10, Malgouyres and Bertrand define a continuous analogue $\Sigma_{S}$ for any near strong 26-surface $S$ which is, in fact, a triangulation of a surface embedded in $\mathbb{R}^{3}$. Hence, to prove that $S$ is an $f^{B M}$-surface it will be enough to show that the simplicial analogue $\mathcal{A}_{S}$ of $S$ is a subdivision of $\Sigma_{S}$. For this we recall more notations and results from [10] in the following paragraphs.

An elementary cube is a closed unit cube with vertices in $Z^{3}$. Notice that the vertices of an elementary cube $C$ are the centroids of eight 3-cells in $R^{3}$ which share a common 0-cell, and this 0-cell is the center of $C$. Given a digital object $O$, we say that an elementary cube $C$ is maximal with respect to $O$ if $C \cap O \neq \emptyset$ and $C \cap O=C^{\prime} \cap O$ whenever $C \cap O \subseteq C^{\prime} \cap O$ for some other elementary cube $C^{\prime}$. Finally, a maximal elementary cube $C$ is said to be simple if for any 3-cell $\sigma \in C \cap O$ the difference $C \cap \mathbb{Z}^{3}-O$ consists of 3 -cells in 6-components of $N_{26}\left(\sigma ; Z^{3}-O\right)$ which are 6 -adjacent to $\sigma$; otherwise $C$ is said non-simple.

Given a maximal elementary cube $C$ with respect to a near strong 26-surface $S$, the vertices of $C \cap S$ can be arranged in a canonical cycle. If $C$ is simple such a cycle is defined in [10] Lemma 5] as the subgraph induced by the vertices of $C \cap S$ in the $1 d$-adjacency graph of $S$; two 3-cell $\sigma, \tau \in S$ are said to be $1 d$ adjacent if either they are 6-adjacent or they are 18-adjacent and no 3-cell in $S$ is 6 -adjacent to both $\sigma$ and $\tau$. Now assume $C$ is non-simple. Lemma 3 in [10] ensures that, up to symmetries or rotations, the only possible configuration of $C \cap S$ is that in Fig. 2(a), where $\sigma \in S$ and $\tau \in C \cap \mathbb{Z}^{3}-S$ is the only 3-cell in a 6-component of $N_{26}\left(\sigma ; \mathbb{Z}^{3}-S\right)$ other than $A_{1}^{\sigma}$ and $A_{2}^{\sigma}$ in Definition 6 . Moreover, both components $A_{1}^{\sigma}, A_{2}^{\sigma}$ have representatives in the set of 3 -cells $\left(C \cap \mathbb{Z}^{3}\right)-(S \cup\{\tau\})$. Without lost of generality, assume that $\tau_{1}, \tau_{2}$ are in $A_{1}^{\sigma}$ (see Fig. [2(a)). Then, the proofs of Lemmas 6 and 7 in [10] actually show that the eight cells depicted in Fig. 2(a) on the left and right hands of $C$ are all in $Z^{3}-S$. Now, in [10, Lemma 8] a canonical cycle in $C \cap S$ is defined as it is sketched in Fig. 2(b).

By the use of canonical cycles, Malgouyres and Bertrand show in [10, Th. 5] that the simplicial complex $\Sigma_{S}$ is a topological surface in $\mathbb{R}^{3}$. Each triangle $T \in \Sigma_{S}$ has as vertices two successive points of the canonical cycle of $C \cap S$, where $C$ is a maximal elementary cube with respect to $S$ containing $T$; and its 
third vertex is either the center of the common 2-face of $C$ and $C^{\prime}$, if there exists a maximal elementary cube $C^{\prime} \neq C$ such that $C^{\prime} \cap S=C \cap S$, or the center of $C$ otherwise.

In order to compare $\Sigma_{S}$ with the simplicial analogue $\mathcal{A}_{S}$ we need the following lemmas.

Lemma 10. Let $O$ be a digital object in $R^{3}$ and $C$ an elementary cube such that $C \cap O \neq \emptyset$. The following two properties are then equivalent.

1. If $C^{\prime}$ is another elementary cube such that $C \cap O \subseteq C^{\prime} \cap O$, then $C^{\prime}=C$.

2. If $\alpha \in R^{3}$ is the 0 -cell in the center of $C$, then $\alpha \in \operatorname{supp}(C \cap O)$.

In particular, if any of these conditions holds, the elementary cube $C$ is maximal with respect to $O$ and $c(\alpha) \in \mathcal{A}_{O}$.

The proof of Lemma 10 is immediate from the definitions of elementary cube and the support of an object. We use also the next lemma concerning centroids of 1-cells.

Lemma 11. Let $S$ be a near strong 26-surface and $\alpha$ an 1-cell in $R^{3}$. Then $c(\alpha) \in \mathcal{A}_{S}$ if and only if the two elementary cubes $C_{1}, C_{2}$ which meet in the 2-face whose center is $c(\alpha)$ are both maximal with respect to $S$ and, moreover, one of the two following conditions holds:

1. $C_{1} \cap S=C_{2} \cap S=s t_{3}\left(\alpha ; R^{3}\right)$

2. st $_{3}(\alpha ; S)=\{\sigma, \tau\}$ and $\sigma, \tau$ are successive in the canonical cycles of both $C_{1} \cap S$ and $C_{2} \cap S$.

Proof. Assume $c(\alpha) \in \mathcal{A}_{S}$. Then, the definition of $f^{B M}$ yields that one of the following cases occurs: (a) $\operatorname{st}_{3}\left(\alpha ; R^{3}\right) \subseteq S$; (b) $\alpha \in \operatorname{supp}(S)$ and $\operatorname{st}_{3}^{*}(\alpha ; S)=$ $\operatorname{st}_{3}(\alpha ; S) \neq \operatorname{st}_{3}\left(\alpha ; R^{3}\right)$; and, (c) $\alpha \in \operatorname{supp}(S)$ and there exist $\sigma_{1}, \sigma_{2} \in \operatorname{st}_{3}^{*}(\alpha ; S)$ with $\sigma_{1} \cap \sigma_{2}=\emptyset$.

In case (a) both elementary cubes $C_{1}$ and $C_{2}$ are maximal and $C_{1} \cap S=$ $C_{2} \cap S=\operatorname{st}_{3}\left(\alpha ; R^{3}\right)$. Otherwise only one of such cubes is maximal and simple with no canonical cycle in it. Here we use Lemma 3 in [10].

Case (b) cannot occur for a near strong 26-surface $S$ since it implies that $C_{1}$ and $C_{2}$ are maximal and simple, by [10, Lemma 3], with no canonical cycle in them.

Finally, if case (c) occurs then both $C_{1}$ and $C_{2}$ are maximal, by Lemma 10. If, in addition, $\operatorname{st}_{3}(\alpha ; S)$ contains only two 3 -cells, these are $1 d$-adjacent and so they are successive vertices in the canonical cycles of $C_{1} \cap S$ and $C_{2} \cap S$ (being $C_{1}$ and $C_{2}$ simple or not).

We finish the proof by showing that $\operatorname{st}_{3}(\alpha ; S)$ does not contain three 3-cells (four 3-cells is case (a)). Otherwise we have 3-cells $\sigma, \tau, \rho \in S$ and $\delta \notin S$ as in Fig. 3. By property (3) in Definition [6 $\rho$ must be 26-adjacent to both 6 -components $A_{1}^{\sigma}$ and $A_{2}^{\sigma}$ which are 6 -adjacent to $\sigma$. From this one readily derives that $x \in S$ or $y, y^{\prime} \in S$. As both $C_{1}$ and $C_{2}$ are maximal and simple, $\delta$ belongs to either $A_{1}^{\tau}$ or $A_{2}^{\tau}$, and hence $\mu \notin S$; here we use Lemma 3 in [10]. Now, we claim that $\mu_{1}, \mu_{2} \notin S$. Otherwise, if $\mu_{1} \in S$ one applies property (3) in Definition 6 to $\mu_{1}$ and $\rho$ and gets that $\mu_{2} \in S$. Hence, $\delta \notin A_{1}^{\tau} \cup A_{2}^{\tau}$, which is a 


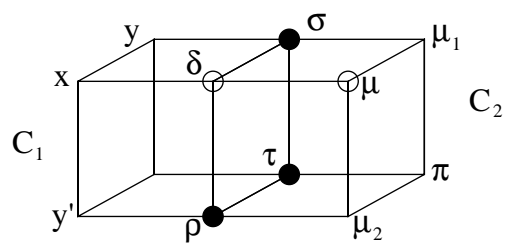

Fig. 3. The center of a 2 -face of an elementary cube which has exactly three of its vertices in a near strong 26-surface $S$ is not lighted for $S$.

contradiction; and similarly for $\mu_{2} \in S$. Finally, $\pi \in S$ by hypothesis and there is no canonical cycle in $C_{2} \cap S$.

Conversely, it is straightforwardly proved that condition (1) in the hypothesis implies case (a), and condition (2) together with the maximality of $C_{1}$ and $C_{2}$ yields case (c).

We are now ready to prove

Proposition 12. Let $S$ be a near strong 26-surface in $\mathbb{Z}^{3}$. Then, the simplicial analogue $\mathcal{A}_{S}$ of $S$ in $\left(R^{3}, f^{B M}\right)$ is a subdivision of $\Sigma_{S}$. So that, $S$ is an $f^{B M}$-surface.

Proof. To prove this result it is enough to show that: (1) Any triangle of $\Sigma_{S}$ is the union of two triangles of $\mathcal{A}_{S}$; and (2) any simplex in $\mathcal{A}_{S}$ is contained in some triangle of $\Sigma_{S}$.

First we prove (1). By definition of $\Sigma_{S}$, its triangles can be drawn inside an elementary cube in exactly three ways (up to rotations or symmetries) as sketched in Fig. 4. In this figure, black dots are points in $S$, while circles are points not in $S$. Moreover, in Fig. 4(a) the only vertices of the elementary cubes belonging to $S$ are exactly the four black dots, but in Fig. 4(b) and (c) there must exist at least another vertex in $S$ in addition to the two black dots. Now, it readily follows from Lemmas [10] and 11] that the triangles in Fig. [5] are in $\mathcal{A}_{S}$ and subdivide the corresponding triangles of $\Sigma_{S}$.

To prove (2) we first observe, from Lemmas 10,11 and the definition of $f^{B M}$, that all the vertices of $\mathcal{A}_{S}$ belong to $\left|\Sigma_{S}\right|$. We next prove that any triangle $\alpha \in \mathcal{A}_{S}$ with vertices in centroids of cells $\alpha_{1}<\alpha_{2}<\alpha_{3}$, is contained in $\left|\Sigma_{S}\right|$.

If $\operatorname{dim} \alpha_{3}=2$, and thus $\operatorname{dim} \alpha_{1}=0$ and $\operatorname{dim} \alpha_{2}=1$ then, Lemmas 10, 11 and the definition of $f^{B M}$ yield that the elementary cube with center $c\left(\alpha_{1}\right)$ is maximal and simple with no canonical cycle in it; here we also use Lemma 3 in 10. Thus, this case cannot occur, and hence there are no tetrahedrons in $\mathcal{A}_{S}$. Therefore $\operatorname{dim} \alpha_{3}=3$, and $\alpha$ lie inside an elementary cube (up to rotations or symmetries) in the three patterns depicted in Fig. 5] Moreover it is readily checked from Lemmas 10 11 and the definition of $f^{B M}$ that the corresponding triangles of Fig. 4 are in $\Sigma_{S}$. Hence (2) holds for triangles. Similarly for edges. 


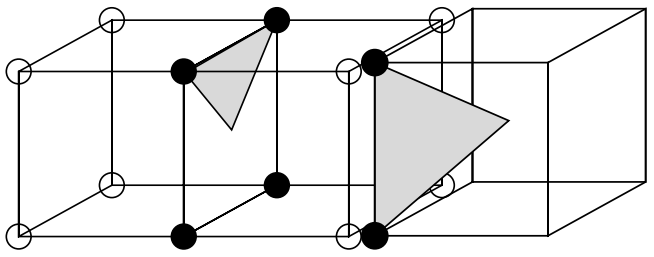

(a)

(b)

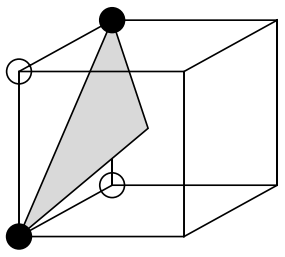

(c)

Fig. 4. The triangles of $\Sigma_{S}$.

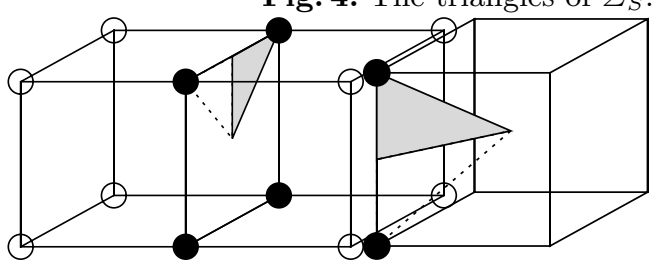

(a)

(b)

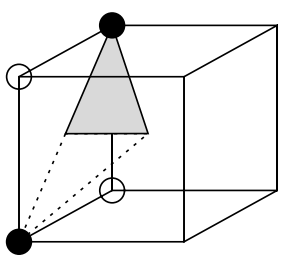

(c)

Fig. 5. The triangles of $\mathcal{A}_{S}$.

\section{$5 \quad f^{B M}$-surfaces are strong 26-surfaces}

In this section we prove that any given $f^{B M}$-surface $S$ in $R^{3}$ is a strongly separating set, and moreover, the four properties in Definition 6 hold for all $\sigma \in S$. So that, by Theorem 7, $S$ is a strong 26-surface. In this proof we use the following properties of $f^{B M}$.

Remark 13. Let $O$ be a digital object in $\left(R^{3}, f^{B M}\right)$ and let $\alpha<\sigma \in O$ with $f^{B M}(O, \alpha)=0$. If $\operatorname{dim} \alpha=2$ then $\alpha=\sigma \cap \tau$ with $\tau$ a 3-cell in $\operatorname{cell}_{3}\left(R^{3}\right)-O$. Moreover if $\operatorname{dim} \alpha=0$ then the definition of $f^{B M}$ yields that $\alpha \notin \operatorname{supp}(O)$, and from Lemma 10 it follows that the elementary cube $C$ with center in $\alpha=c(\alpha)$ contains a 2 -face whose four vertices are (the centroids of) 3 -cells in $\operatorname{cell}_{3}\left(R^{3}\right)-O$. Finally, if $\operatorname{dim} \alpha=1$ then two cases are possible:

Case a: $\alpha \notin \operatorname{supp}(O)$. Then $c(\alpha)$ is the center of a 2 -face $A$ common to two elementary cubes, and the vertices of an edge of $A$ are (centroids of) two 3-cells in $\operatorname{cell}_{3}\left(R^{3}\right)-O$.

Case b: $\alpha \in \operatorname{supp}(O)$. If $\beta_{1}, \beta_{2} \in R^{3}$ are the two 0-cells with $\beta_{1}, \beta_{2}<\alpha$, then the definition of $f^{B M}$ implies that $f^{B M}\left(O, \beta_{i}\right)=1$ and $f^{B M}\left(O, \beta_{j}\right)=0$, where $\{i, j\}=\{1,2\}$.

Lemma 14. Let $O$ be a digital object in $\left(R^{3}, f^{B M}\right)$ and $\alpha<\sigma \in O$. If $c(\alpha) \notin \mathcal{A}_{O}$ then $s_{3}\left(\alpha ; R^{3}\right)-O$ and $G_{6}\left(\sigma ; s t_{3}\left(\alpha ; R^{3}\right)-O\right)$ are respectively contained in $a$ 6 -component of $s t_{3}^{*}\left(\sigma ; R^{3}\right)-O$ and $G_{6}\left(\sigma ; \mathbb{Z}^{3}-O\right)$, which are 6 -adjacent to $\sigma$. Furthermore, $G_{6}\left(\sigma ; s t_{3}\left(\alpha ; R^{3}\right)-O\right)=\emptyset$ if and only if $\operatorname{dim} \alpha=1$ and $c\left(\gamma_{1}\right), c\left(\gamma_{2}\right) \in$ $\mathcal{A}_{O}$, where $\gamma_{1}, \gamma_{2}<\sigma$ are the two 2-cells with $\alpha=\gamma_{1} \cap \gamma_{2}$.

Proof. For $\operatorname{st}_{3}\left(\alpha ; R^{3}\right)-O$ the lemma is immediate from Remark 13 Also Remark 13 directly implies the result for $G_{6}\left(\sigma ; \operatorname{st}_{3}\left(\alpha ; R^{3}\right)-O\right)$ except in the case 
$\operatorname{dim} \alpha=0$, for which it suffices to check the essentially distinct six patterns of $O$ in $\operatorname{st}_{3}^{*}\left(\alpha ; R^{3}\right)$. Moreover, the characterization of $G_{6}\left(\sigma ; \operatorname{st}_{3}\left(\alpha ; R^{3}\right)-O\right)=\emptyset$ is also derived from Remark 13

Another important ingredient in the proofs below is the notion of relative ball in $\left(\mathbb{R}^{n}, M\right)$, where $M \subseteq \mathbb{R}^{n}$ is a combinatorial $(n-1)$-manifold; see 2 . More explicitly, a relative ball in $\left(\mathbb{R}^{n}, M\right)$ is a pair of balls $\left(B^{n}, B^{n-1}\right)$ such that $B^{n-1} \subseteq B^{n}, B^{n-1} \cap \partial B^{n}=\partial B^{n-1}$ and $B^{n} \cap M=B^{n-1}$.

Remark 15. Let $f$ be a w.l.f. on $R^{n}$ with $\left|\mathcal{A}_{R^{n}}\right|=\mathbb{R}^{n}$. Assume that $M$ is a connected digital $(n-1)$-manifold without boundary. Then the classical JordanBrouwer separation theorem states that $\left|\mathcal{A}_{R^{n}}\right|-\left|\mathcal{A}_{M}\right|=\mathbb{R}^{n}-\left|\mathcal{A}_{M}\right|$ has two connected components. Moreover, any relative ball $\left(B^{n}, B^{n-1}\right)$ in $\left(\mathbb{R}^{n},\left|\mathcal{A}_{M}\right|\right)$ verifies that $B^{n}-B^{n-1}$ has exactly two components, each of which is contained in a distinct component of $\mathbb{R}^{n}-\left|\mathcal{A}_{M}\right|$; see [2]. Furthermore, the components of $\mathbb{R}^{n}-\left|\mathcal{A}_{M}\right|$ determine the $M$-components of $\operatorname{cell}_{n}\left(R^{n}\right)-M$ according to Theorem 2

In particular, as $f^{B M}\left(\operatorname{cell}_{3}\left(R^{3}\right), \alpha\right)=1$ for all $\alpha \in R^{3}$, the continuous analogue $\left|\mathcal{A}_{R^{3}}^{f^{B M}}\right|$ is $\mathbb{R}^{3}$. Thus $f^{B M}$-surfaces satisfy a digital Jordan property; that is, if $S$ is an $f^{B M}$-surface then $\operatorname{cell}_{3}\left(R^{3}\right)-S$ has two $S$-components, and, according to Remark 4 two 6-components. Moreover, if $\left(B^{3}, B^{2}\right)$ is a relative ball in $\left(\mathbb{R}^{3},\left|\mathcal{A}_{S}^{f^{B M}}\right|\right)$ then the components of $B^{3}-B^{2}$ determine the 6-components of $\operatorname{cell}_{3}\left(R^{3}\right)-S$.

For the following proposition recall that given a simplicial complex $K$ the star and the link of a vertex $v \in K$ are the sets $\operatorname{st}(v ; K)=\{\sigma \in K ; \sigma \leq \tau \in K, v \in \tau\}$ and $\operatorname{lk}(v ; K)=\{\rho \in \operatorname{st}(v ; K) ; v \notin \rho\}$, respectively.

Proposition 16. Let $f$ be a w.l.f. on $R^{n}$ such that $f(M, \alpha)=1$ whenever $\operatorname{st}_{n}\left(\alpha ; R^{n}\right)$ is contained in the digital object $M$. If $M$ is a digital $(n-1)$-manifold in $\left(R^{n}, f\right)$ then the pair $\left(\left|\operatorname{st}\left(c(\alpha) ; \mathcal{A}_{R^{n}}\right)\right|,\left|\operatorname{st}\left(c(\alpha) ; \mathcal{A}_{M}\right)\right|\right)$ is a relative ball in $\left(\mathbb{R}^{n},\left|\mathcal{A}_{M}\right|\right)$ for each cell $\alpha \in R^{n}$ with $c(\alpha) \in \mathcal{A}_{M}$.

Proof. It is readily derived from the hypothesis that $\left|\mathcal{A}_{R^{n}}\right|=\mathbb{R}^{n}$. Then, as $\left|\mathcal{A}_{M}\right|$ is a combinatorial $(n-1)$-manifold without boundary, $\operatorname{st}\left(c(\alpha) ; \mathcal{A}_{M}\right) \subseteq$ $\operatorname{st}\left(c(\alpha) ; \mathcal{A}_{R^{n}}\right)$ are balls of the corresponding dimensions. In fact, $\operatorname{st}\left(c(\alpha) ; \mathcal{A}_{M}\right)=$ $\operatorname{st}\left(c(\alpha) ; \mathcal{A}_{R^{n}}\right) \cap \mathcal{A}_{M}$ since $\mathcal{A}_{M} \subseteq \mathcal{A}_{R^{n}}$ is a full subcomplex. The same argument yields $\operatorname{lk}\left(c(\alpha) ; \mathcal{A}_{M}\right)=\operatorname{lk}\left(c(\alpha) ; \mathcal{A}_{R^{n}}\right) \cap \mathcal{A}_{M}$. Notice that $\operatorname{lk}\left(c(\alpha) ; \mathcal{A}_{M}\right)$ is the boundary of $\operatorname{st}\left(c(\alpha) ; \mathcal{A}_{M}\right)$.

Remark 17. According to Remark 15, $D_{\alpha}=\left|\operatorname{st}\left(c(\alpha) ; \mathcal{A}_{R^{n}}\right)\right|-\left|\operatorname{st}\left(c(\alpha) ; \mathcal{A}_{M}\right)\right|$ has two components. Moreover, it is easy to prove that for a component $C$ there always exists a cell $\beta \in R^{n}$ with $c(\beta) \in C$. For this one uses that $\mathcal{A}_{M}$ is full in $\mathcal{A}_{R^{n}}$.

Proposition 18. Any connected digital $f^{B M}$-surface $S$ in $\left(R^{3}, f^{B M}\right)$ is strongly separating. 
Proof. Since $S$ is an $f^{B M}$-surface we know, by Remark 15, that $Z^{3}-S=$ $\operatorname{cell}_{3}\left(R^{3}\right)-S$ has two 6-components. So, it suffices to check that each $\sigma \in S$ is 6 -adjacent to both of them. By Remark 15 , one also knows that the 6-components of $Z^{3}-S$ are determined by the (continuous) components $C_{1}, C_{2}$ of $D_{\sigma}=$ $\left|\operatorname{st}\left(c(\sigma) ; \mathcal{A}_{R^{3}}\right)\right|-\left|\operatorname{st}\left(c(\sigma) ; \mathcal{A}_{S}\right)\right|$; see Proposition 16. Then, the result follows from the existence of $c\left(\alpha_{i}\right) \in C_{i}, i=1,2$ (see Remark 17) and Lemma 14

Proposition 19. Any connected digital $f^{B M}$-surface $S$ in $\left(R^{3}, f^{B M}\right)$ is a near strong 26-surface

Proof. It will suffice to prove that properties (1)-(4) in Definition 6 hold for each $\sigma \in S$.

Property (1) will follow if we prove that $N_{26}\left(\sigma ; Z^{3}-S\right)=\operatorname{st}_{3}^{*}\left(\sigma ; R^{3}\right)-S$ has at most two 6 -components which are 6 -adjacent to $\sigma$, since it has at least two as $S$ is strongly separating. And this follows by showing that if $\sigma_{1}, \sigma_{2} \notin S$ are 6 -adjacent to $\sigma$, the existence of a path $c\left(\sigma \cap \sigma_{1}\right)=c\left(\gamma_{0}\right), \ldots, c\left(\gamma_{k}\right)=$ $c\left(\sigma \cap \sigma_{2}\right)$ in the difference $D_{\sigma}=\left|\operatorname{st}\left(c(\sigma) ; \mathcal{A}_{R^{3}}\right)\right|-\left|\operatorname{st}\left(c(\sigma) ; \mathcal{A}_{S}\right)\right|$ implies that $\sigma_{1}$ and $\sigma_{2}$ are in the same 6 -component of $N_{26}\left(\sigma ; Z^{3}-S\right)$. Without lost of generality assume $\gamma_{i}<\gamma_{i-1}$. Then $\operatorname{st}_{3}\left(\gamma_{i-1} ; R^{3}\right)-S \subseteq \operatorname{st}_{3}\left(\gamma_{i} ; R^{3}\right)-S$ and moreover they are non-empty sets since $f^{B M}$ satisfies $f^{B M}(O, \alpha)=1$ whenever $\operatorname{st}_{3}\left(\alpha ; R^{3}\right) \subseteq O$. Then Lemma 14 and an inductive argument on $k$ show that $\left\{\sigma_{1}, \sigma_{2}\right\} \subseteq \cup_{i=0}^{k} \operatorname{st}_{3}\left(\gamma_{i} ; R^{3}\right)-S$ is contained in some 6 -component of $N_{26}\left(\sigma ; Z^{3}-\right.$ $S)$ which is 6 -adjacent to $\sigma$.

To prove property (2) it will suffice to check that $G_{6}\left(\sigma ; \mathbb{Z}^{3}-S\right)$ contains at most two 6-components, since it has at least two by property (1). This is proved following the same pattern as for the proof of property (1) above if we show that $G_{6}\left(\operatorname{st}_{3}\left(\gamma_{i} ; R^{3}\right)-S\right)$ are non-empty sets, which is readily derived from Lemma 14 .

For the proof of property (4), let us consider the two 6-components $A_{1}^{\sigma}$ and $A_{2}^{\sigma}$ of $N_{26}\left(\sigma ; Z^{3}-S\right)$ which are 6 -adjacent to $\sigma$. Then, it is not difficult to prove that the two 6 -components of $G_{6}\left(\sigma ; Z^{3}-S\right)$ are $G_{6}\left(\sigma ; A_{i}^{\sigma}\right), i=1,2$. So, property (4) will follow by proving that any $\tau \in N_{6}(\sigma ; S)$ is 6 -adjacent to both $G_{6}\left(\sigma ; A_{1}^{\sigma}\right)$ and $G_{6}\left(\sigma ; A_{2}^{\sigma}\right)$. For this we consider the 2-cell $\gamma=\sigma \cap \tau$ (notice that $\left.c(\gamma) \in \mathcal{A}_{S}\right)$ then one observes that any centroid $c(\alpha)$ in the difference $D_{\gamma}$ also belongs to $D_{\sigma}$. As the components of both $D_{\gamma}$ and $D_{\sigma}$ determine the components of $\left|\mathcal{A}_{R^{3}}\right|-\left|\mathcal{A}_{S}\right|$ then two cells $\alpha_{1}, \alpha_{2}<\gamma$ can be found with $c\left(\alpha_{i}\right) \in C_{i}$, where $C_{i}(i=1,2)$ are the components of $D_{\sigma}$. Notice also that the proof of property (1) above actually shows that $C_{1}$ and $C_{2}$ determine the two 6-components $A_{1}^{\sigma}$, $A_{2}^{\sigma}$. In fact, for a cell $\alpha \in R^{3}, c(\alpha) \in C_{i}$ if and only if $\operatorname{st}_{3}\left(\alpha ; R^{3}\right)-S \subseteq A_{i}^{\sigma}$. Now Remark 13 ensures that if $\operatorname{dim} \alpha_{i}=0$ or $\operatorname{dim} \alpha_{i}=1$ and $\alpha_{i} \notin \operatorname{supp}(S)$ then there exists a 3 -cell in $G_{6}\left(\sigma ; \operatorname{st}_{3}\left(\alpha_{i} ; R^{3}\right)-S\right)$ which is 6 -adjacent to $\tau$. Otherwise, if $\operatorname{dim} \alpha_{i}=1$ and $\alpha_{i} \in \operatorname{supp}(S)$, from Remark 13 there is a 0-cell $\beta<\alpha$ with $f^{B M}(S, \beta)=0$ and one applies the previous case to $\beta$.

Finally we prove property (3). From the definition of $f^{B M}$ we first derive that for any $\tau \in N_{26}(\sigma ; S)$ there exists $\alpha_{\tau}<\tau \cap \sigma$ with $c\left(\alpha_{\tau}\right) \in \partial \operatorname{st}\left(c(\sigma) ; \mathcal{A}_{S}\right)$. If $\operatorname{dim} \alpha_{\tau}=2$ the proof above of property (4) yields the result. Otherwise, if $\operatorname{dim} \alpha_{\tau}<2$, one finds a centroid $c\left(\alpha_{i}\right)$ in each component $C_{i} \subseteq D_{\sigma}(i=1,2)$ 
such that $\alpha_{i}<\alpha_{\tau}$ or $\alpha_{\tau}<\alpha_{i}$. In any case all 3-cells in $\operatorname{st}_{3}\left(\alpha_{i} ; R^{3}\right)-S \subseteq A_{i}^{\sigma}$ are 26-adjacent to $\tau$. Here we use again the fact that $f^{B M}(S, \alpha)=1$ whenever $\operatorname{st}_{n}\left(\alpha ; R^{3}\right) \subseteq S$.

\section{Acknowledgements:}

The authors wish to thank the reviewers for their valuable comments to improve the final version of this paper.

This work has been partially supported by the project DGICYT PB96-1374.

\section{References}

1. R. Ayala, E. Domínguez, A.R. Francés, A.Quintero, J. Rubio. On surfaces in digital topology. Proc. of the 5th Workshop on Discrete Geometry for Computer Imagery DGCI'95. (1995) 271-276.

2. R. Ayala, E. Domínguez, A.R. Francés, A.Quintero. Determining the components of the complement of a digital $(n-1)$-manifold in $\mathbb{Z}^{n}$. Lecture Notes in Computer Science. 1176(1996) 163-176.

3. R. Ayala, E. Domínguez, A.R. Francés, A.Quintero. Digital Lighting Functions. Lecture Notes in Computer Science. 1347(1997) 139-150.

4. G. Bertrand, M. Couprie. Some structural properties of discrete surfaces. Lecture Notes in Computer Science. 1347(1997) 113-124.

5. G. Bertrand, R. Malgouyres. Some topological properties of discrete surfaces. Lecture Notes in Computer Science. 1176(1996) 325-336.

6. E. Khalimsky, R. Kopperman, P.R. Meyer. Computer Graphics and connected topologies on finite ordered sets. Topology and its Applications. 36(1990) 1-17.

7. T.Y. Kong, A.W. Roscoe. Continuous Analogs of Axiomatized Digital Surfaces. Computer Vision, Graphics, and Image Processing. 29(1985) 60-86.

8. T.Y. Kong, A. Rosenfeld. Digital Topology: Introduction and Survey. Computer Vision, Graphics, and Image Processing. 48(1989) 357-393.

9. V.A. Kovalevsky. Finite topology as applied to image analysis. Computer Vision, Graphics, and Image Processing. 46(1989) 141-161.

10. R. Malgouyres, G. Bertrand. Complete local characterization of strong 26-surfaces: Continuous analogs for strong 26-surfaces. Int. Jour. on Pattern Recognition and Artificial Intelligence. Special issue on Parallel Image Analysis. To appear.

11. D.G. Morgenthaler, A. Rosenfeld. Surfaces in Three-Dimensional Digital Images. Information and Control. 51(1981) 227-247.

12. C.P. Rourke, B.J. Sanderson. Introduction to Piecewise linear topology. Ergebnisse der Math., 69. Springer, 1972. 